\title{
An EEG-based Brain Mapping to Determine Mirror Neuron System in Patients with Chronic Stroke during Action Observation
}

\author{
Eun-Ju Kuk', Jong-man Kim² \\ 'Department of Physical Therapy, Division of Health, Seonam University, Namwon; ${ }^{2}$ Department of Occupational Therapy, Division of Health, Jeonju \\ University, Jeonju, Korea
}

Purpose: The aim of this study was to compare EEG topographical maps in patients with chronic stroke after action observation physical training.

Methods: Ten subjects were recruited from a medical hospital. Participants observed the action of transferring a small block from one box to another for 6 sessions of 1 minute each, and then performed the observed action for 3 minutes, 6 times. An EEG-based brain mapping system with 32 scalp sites was used to determine cortical reorganization in the regions of interest (ROIs) during observation of movement. The EEG-based brain mapping was comparison in within-group before and after training. ROls included the primary sensorimotor cortex, premotor cortex, superior parietal lobule, inferior parietal lobule, superior temporal lobe, and visual cortex. EEG data were analyzed with an average log ratio in order to control the variability of the absolute mu power. The mu power log ratio was in withingroup comparison with paired t-tests.

Results: Participants showed activation prior to the intervention in all of the cerebral cortex, whereas the inferior frontal gyrus, superior frontal gyrus, precentral gyrus, and inferior parietal cortex were selectively activated after the training. There were no differences in mu power between each session.

Conclusion: These findings suggest that action observation physical training contributes to attaining brain reorganization and improving brain functionality, as part of rehabilitation and intervention programs.

Keywords: Reorganization, Stroke, EEG, Brain mapping, Mirror neurons

\section{INTRODUCTION}

Most of the activities of daily living are performed through the functional movements of the arms and the hands. Since stroke patients do not use their paralyzed arms and hands, their muscular strength is reduced, spasticity increases, and the reduction or pain of the soft tissues is caused. ${ }^{1}$ These problems limit the activities of daily living, and among these limitations, there occur numerous problems of hand dexterity.

As effective interventional methods that recover a chronic stroke patient clinically, the techniques that directly cause movement to increase such as the constraint induced movement therapy (CIMT),

Received May 26, 2015 Revised Jun 14, 2015

Accepted Jun 18, 2015

Corresponding author Jong-man Kim

E-mail paulkjm66@hanmail.net robot-assisted training and dance sports in virtual reality, or transcutaneous electrical nerve stimulation (TENS) and interferential current therapy (ICT) are applied. ${ }^{2-6}$ However, it has been reported that it is difficult to provide experience-dependent inputs to the patients with low exercise functions?

The method used to overcome such a limitation is the priming technique. ${ }^{8}$ The priming technique increases the excitability of the motor system that was damaged by stroke in order to enhance the re-formation of a brain. The intervention that makes the sensory motor systems prepare for the purpose of exercise practice includes the imaging training, action observation training (AOT), repetitive transcranial magnetic stimulation, and transcranial direct current

Copylight (C2015 The Korea Society of Physical Therapy

This is an Open Access article distribute under the terms of the Creative Commons Attribution Non-commercial License (Http:// creativecommons.org/license/by-nc/3.o.) which permits unrestricted non-commercial use, distribution, and reproduction in any medium, provided the original work is properly cited. 
stimulation, among others. Of these, the method that can be applied easily at a low cost is AOT.

AOT is a method that activates one of the frontoparietal circuits that prepare and control the purpose-oriented action according to the sight and body control by observing the exercise tasks carried out by another person. ${ }^{9}$ This circuit not only is activated not only when it moves by itself but also when it observes another person move. Due to this characteristic, this circuit is called the mirror neuron system (MNS). ${ }^{10}$

As brain imaging techniques have become more developed, there has been more research that checks MNS by using EEG. In EEG, $\mu$ rhythm is measured- $\mu$ rhythm which is the frequency of $8-13 \mathrm{~Hz}$ occurring in the exercise area during a rest time. While actions are executed and observed, $\mu$ rhythm decreases. While EEG has a high timely resolution, it has a low spatial resolution. To compensate for this limitation, the methods are developed that presume the nerve activation location through measured EEG materials. ${ }^{11,12}$ Im et al. ${ }^{13}$ suggested the possibility of a real-time cortex rhythm activation observation system based on EEG materials so that an immediate cortex rhythm can be visualized.

After the MNS was discovered, many researchers attempted to discover its function, and suggest that MNS performs an interventional role that understands and sympathizes the meaning of an observed motion. In addition, they found that MNS is activated while it prepares in order to imitate motions. ${ }^{14}$ In this way, since an observer can make the symbol of a motion on MNS and an observed motion agreed, this is called the observation-execution matching. The observation-execution matching is an important mechanism related to recognizing and understanding motions.

Nevertheless, although there has been much research thus far that observed the activation of MNS by using EEG, the research on how the activation of MNS is changed during a training is insufficient. Thus, this study was conducted to determine if a training that involves the observation of motions and repeated physical trainings affects the activation of the MNS.

\section{METHODS}

\section{Subjects}

The chronic stroke patient subjects who participated in the training were 11 selected hemiplegia patients who were diagnosed with stroke and had been treated in a rehabilitation hospital. During the training, the EEGs of 10 patient were analyzed, after 1 patient who failed to concentrate was excluded. All subjects listened to the research purpose and training procedure and voluntarily signed on the consent forms before measurement. The subjects were selected from the group of patients who had been diagnosed with stroke more than six months earlier, who had no other neurological lesions before the occurrence of stroke, who had a MMSE-K score higher than 24 points, who had no cognitive-perceptual disorders and could follow instructions, who had no visual or hearing problems and no aphasia, who could hold a block of $5 \times 5 \mathrm{~cm}$ with a paralyzed hand, and who had been receiving physical therapy and occupational therapy for the past three months.

\section{Experimental methods}

\section{1) Measurement}

\section{(1) Collection of EEG materials}

The EEG was measured when a motion is observed using the WEEG-32 (LXE3232-RF, LAXTHA Inc., Korea). The brain wave of the subject was saved in a computer through $256 \mathrm{~Hz}$ sampling frequency, pass filter of $0.5-50 \mathrm{~Hz}$, and 12-bit AD conversion. The EEG materials were collected by 32 electrodes that were attached to the head. The electrodes were attached according to the extended 10-20 method of electrode placement, and the reference electrodes with a diameter of $45 \mathrm{~mm}$ were attached to the back sides of the both ears. The electrodes used are dish formed disc electrodes coated with gold, and they were attached using electrode paste that is used only for EEG. In addition, gauges were covered on the attached electrodes so that the electrode paste did not harden rapidly and could be fixed on the surface of the head.

After electrodes were attached, the subjects sat in comfortable chairs in an inspection room that was free of noise and watched the 27 inch LED monitor that was $2 \mathrm{~m}$ away. The measurement was performed in a silent environment in a state in which the physical movements of the subjects were controlled. In order to minimize their movements, subjects were requested to rest their arms comfortably on their thighs. In order to induce the subjects to concentrate during the observation of movements, they were told that they would be questioned about their movements when measurement is finished. 


\section{(2) Processing of EEG materials}

The EEGs were measured for 20 seconds in each condition, with the first and last five seconds removed in order to remove the artifacts that can occur when the video starts and ends. This study used the $\mu$ rhythm obtained from $\mathrm{C} 3$ and $\mathrm{C} 4$, which correspond to the areas of the sensorymotor cortex, for analysis.

In order to adjust the variability occurring due to personal differences such as the thickness of head bone, electrode arrangement, and resistance, Telescan 3.08 program (LAXTHA Inc., Korea) was used.

\section{(3) Brain mapping system based on EEG}

In order to evaluate the change of the cortex electricity activation of the brain known as the mirror neuron system, the event-related spectral perturbation (ERSP) that enables the measurement of average changes of the spectrum power was used. ERSP analysis was performed by EEGLAB. In each condition, the 20 second-part from which the first and last five seconds of the video were removed was extracted for the EEG analysis. The $\mu$ frequency band $(8-12 \mathrm{~Hz}) \mathrm{re}-$ lated to the movement of the limbs was used to show a brain map. The brain map was represented by evaluating the spatial changes in the areas where electrodes were attached while the motions were observed before and after training.

\section{2) Design and procedure of study}

The EEGs were measured by showing a box for the purpose of hand dexterity and a motion picture equal to the wood block examination to the action observation training group. The subjects repeated this process of observing the video for 1 minute, and receiving training for 3 minutes six times.

In order to check EEG changes before and after the training, the EEGs were measured while the motions were observed. As one of the subject slept to not concentrate while the video were observed, only the materials of 10 subjects were collected. Participants were asked about observed action.

\section{3) Training video}

The video provided to the action observation training group showed the motion that moves a wood block. The video used for training was produced with the motion that moves a wood block with the right hand and the motion that moves a wood block with the left hand. The time of the motion was 30 seconds for the right hand and 30 seconds for the left hand.

\section{4) Statistical analysis}

The general characteristics of the subjects were obtained using descriptive statistics. In order to check the change of $\mu$ rhythm before and after the training of the subjects, the paired t test was performed. The SPSS version 17.0 for Windows, a statistical software package, was used for all analyses. Descriptive analysis was used to examine the EEG-based brain map.

\section{RESULTS}

\section{General characteristics of study subjects}

A total of 10 subjects participated in the training, and their general characteristics are as shown (Table 1).

\section{Comparison of $\mu$ power $\log$ ratios}

In order to find the difference of $\mu$ power $\log$ ratios while motions were observed before and after the training of the subjects, the mean values of the absolute power log ratio were produced and compared (Table 2). There was no significant difference between the times before and after training $(\mathrm{p}>0.05)$.

\section{Comparison of brain maps between times}

The results of brain maps before and after the action observation training were shown (Figure 1). Before training, activation occurred

Table 1. General characteristic

\begin{tabular}{lcc}
\hline & Division/Unit & Experiment group $(n=10)$ \\
\hline Gender & Male/Female & $4 / 6$ \\
Dominant hand & Left/Right & $0 / 10$ \\
Type & Infarction/Hemorrhage & $5 / 5$ \\
Paretic side & Left/Right & $4 / 6$ \\
Age & Age & $60.00 \pm 9.36^{\star}$ \\
Onset & Month & $15.30 \pm 6.77$ \\
MMSE-K & Score & $25.00 \pm 1.63$ \\
\hline
\end{tabular}

${ }^{*}$ Mean \pm Standard Deviation

Table 2. Comparison pre-test and post-test of mu power

\begin{tabular}{ccccc}
\hline & Pre-test & Post-test & $t$ & $p$ \\
\hline AOT group & $-0.04 \pm 0.06^{*}$ & $-0.03 \pm 0.05$ & -0.34 & 0.743 \\
\hline
\end{tabular}

*Mean \pm Standard Deviation

AOT, action observation training. 


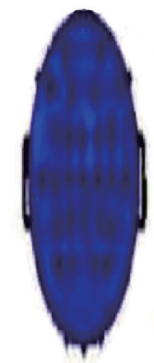

Before training

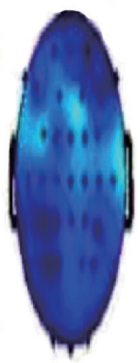

After training
Figure 1. Brain maps before and after training.

in the superior frontal gyrus, middle frontal gyrus, inferior frontal gyrus, precentral gyrus, postcentral gyrus, and inferior parietal cortex. After training, activation occurred in the superior frontal gyrus, inferior frontal gyrus, precentral gyrus, postcentral gyrus, and inferior parietal cortex (Table 3).

\section{DISCUSSION}

The function of the upper limbs of stroke patients is one of the most basic elements in the performance of the activities of daily living, and achieving functional upper limbs functions is an important aim in rehabilitation. In this study, the action observation physical training (AOT) was applied to chronic stroke patients 6 times to produce the effect on the activation of MNS by the brain mapping system based on EEG. As a result, although there was no change of $\mu$ rhythm between times, MNS showed selective activation after AOT.

In order to increase the hand functions of chronic stroke patients, the task-specific training should be repeated. ${ }^{15}$ In addition, the application of a method that increases the reversibility of the neural network can increase hand functions. ${ }^{8}$

The imitation of an observed motion is a cognitive task that does not require excessive efforts. ${ }^{16}$ Iacoboni et al. ${ }^{17}$ reported that the IFG cortex, front parietal lobe area, parietal lobe cortex, and STS area were activated in the conditions in which the targeted motions were observed using fMRI. In addition, researchers argued that this result is equal to the MNS that is activated while the basic circuit involving imitation observes.

It is impossible to know in which area of the brain there is a difference using EEG materials. In order to complement such a lower
Table 3. Activation of mirror neuron system areas

\begin{tabular}{lll}
\hline \multicolumn{1}{c}{ Pre-test } & \multicolumn{1}{c}{ Post-test } \\
\hline AOT group & Superior frontal gyrus (BA*6,9) & Superior frontal gyrus (BA6) \\
& Middle frontal gyrus (BA46) & Inferior frontal gyrus (BA45) \\
Inferior frontal gyrus (BA45) & Precentral gyrus (BA4) \\
Precentral gyrus (BA4) & Postcentral gyrus (BA1,2,3) \\
Postcentral gyrus (BA1,2,3) & Inferior parietal cortex (BA7,40) \\
Inferior parietal cortex (BA7,40) & \\
\hline *Brodmann area. & \\
AOT, action observation training.
\end{tabular}

spatial resolution, a brain mapping system was developed that can measure the brain location based on EEG materials. ${ }^{13}$ The locations of EEG electrodes attached to the subjects in this study were the regions of interest (ROI) about MNS based on the research findings of Koessler et al..$^{18}$ Before training, it was confirmed that in all locations of electrodes, MNS was activated, and after training, it could be verified that MNS was activated in the superior frontal gyrus, precentral gyrus, postcentral gyrus, and inferior parietal cortex. Park et al. ${ }^{19}$ reported that as a result of confirming the reorganization of the cerebrum through a training, the activation of the prefrontal area that was shown before the training was not activated after a shortterm training. Authors insisted that the short-term intensive training led to a learning, which decreased the cognitive use when the task was performed, causing the activation of the unnecessary brain area to disappear. The authors of this study also suggested that by repeating the trainings of 6 times, the subjects went through a learning process to cause the selective activation of MNS to occur compared to the time prior to the training.

The activation of the right inferior parietal cortex decreases due to the adaptation of fMRI if the same motions continue to be repeated. ${ }^{20}$ In this study, during the training, the non-activation of the right inferior parietal cortex was gradually shown. In addition, the non-activation of the right inferior parietal cortex was also shown after the training. This is considered to indicate that the activation of the right inferior parietal cortex tends to decrease while, by repetitively observing and conducting the same motion, the training is performed in the same way as that in which the training of the result of the prior research was performed.

The results of this study may prove the positive effects that decrease the unnecessary cognitive action of task performance during AOT and activate the MNS of the needed areas. However, the influence of AOT in patients with more sever motor impairment has not 
been investigated. And it is necessary to conduct research that will confirm the change of $\mu$ rhythm by applying the AOT of a long term.

\section{REFERENCES}

1. Gjelsvik BEB. The bobath concept in adult neurology, Reinheim-Zeilhand, Germany, 2008.

2. Yang SH, Lee YH, Lee KS. The effects of modified constraint-induced movement therapy and bilateral arm training on the upper extremity performance of individuals with chronic hemiparetic stroke. J Kor Phy Ther. 2011;23(5):65-72.

3. Lee DS, Lee KH, Kang TW, et al. Effect of early robot-assisted training using virtually reality program in patient with stroke. J Kor Phy Ther. 2013;25(4):195-203.

4. Kim WN, Lee KY. Effects of dance sports in virtual reality on balance, depression and ADL in stroke patients. J Kor Phy Ther. 2013;25(5):360-5.

5. Byun DU, Shin WS. Effects of transcutaneous electrical nerve stimulation depending on frequency. J Kor Phy Ther. 2013;25(3):136-42.

6. Kim BY, Choi WH. The effects of interferential current therapy on spasticity, range of motion, and balance ability in stroke patient. J Kor Phy Ther. 2013;25(4):187-94.

7. Taub E, Uswatte G, Morris DM. Improved motor recovery after stroke and massive cortical reorganization following constraint-induced movement therapy. Phys Med Rehabil Clin N Am. 2003;14(1):77-91.

8. Pomeroy V, Aglioti SM, Mark VW, et al. Neurological principles and rehabilitation of action disorders: rehabilitation interventions. Neurorehabil Neural Repair. 2011;25(5):33-43.
9. Frey SH, Fogassi L, Grafton S, et al. Neurological principles and rehabilitation of action disorders: computation, anatomy, and physiology (CAP) model. Neurorehabil Neural Repair. 2011;25(5):6-20.

10. Iacoboni M, Molnar-Szakacs I, Gallese V, et al. Grasping the intentions of others with one's own mirror neuron system, PLoS Biol. 2005;3(3):79.

11. Koles ZJ. Trends in EEG source localization. Electroencephalogr Clin Neurophysiol. 1998;106:127-37.

12. Mosher JC, Leahy RM, Lewis PS. EEG and MEG: forward solutions for inverse solutions. IEEE Trans Biomed Eng. 1999;46:245-59.

13. Im CH, Hwang HJ, Che H, et al. An EEG-based real-time cortical rhythmic activity monitoring system, Physiol Meas. 2007;28:1101-13.

14. Krams M, Rushworth MFS, Deiber MP, et al. The preparation, execution, and suppression of copied movements in the human brain. Exp Brain Res. 1998;120:386-98.

15. Carr JH, Shepherd RB. Neurological rehabilitation: optimizing motor performance, 2nd ed, Edinburgh, New York, 2010.

16. Buccino G, Solodkin A, Small SL. Functions of the mirror neuron system: implications for neurorehabilitation. Cog Behav Neurol. 2006; 19:55-63.

17. Iacoboni M, Woods RP, Brass M, et al. Cortical mechanisms of human imitation. Science. 1999;286:2526-8.

18. Koessler L, Maillard L, Benhadid A et al. Automated cortical projection of EEG sensors: anatomical correlation via the international 10-10 system. NeuroImage. 2009;46(1):64-72.

19. Park JW, Kim JM, Seo JH, et al. Reorganization of motor network and the effect of cross education derived from unilateral coordination training. Phys Ther Korea. 2002;9(3):67-76.

20. Chong TT, Cunnington R, Williams MA, et al. fMRI adaptation reveals mirror neurons in human inferior parietal cortex. Current Biology. 2008;18:1576-80. 